\title{
Bounded solutions for the nonlinear wave equation
}

\author{
Tacksun Jung ${ }^{1}$ and Q-Heung Choi ${ }^{2 *}$
}

${ }^{\text {"Correspondence: }}$

qheung@inha.ac.kr

${ }^{2}$ Department of Mathematics

Education, Inha University, Incheon,

402-751, Korea

Full list of author information is

available at the end of the article

\begin{abstract}
We investigate the number of periodic weak solutions for the wave equation with nonlinearity decaying at the origin. We get a theorem which shows the existence of a bounded weak solution for this problem. We obtain this result by approaching the variational method and applying the critical point theory for the indefinite functional induced from the invariant subspaces and the invariant functional.
\end{abstract}

MSC: 35L05; 35L70

Keywords: wave equation; critical point theory; invariant function; invariant subspace; (P.S.) c condition; eigenvalue problem

\section{Introduction and statement of the main result}

Let $g(x, t, u)$ be a $C^{1}$ function from $[0, \pi] \times R \times R$ to $R$ and $T$-periodic in $t$. In this paper we are concerned with the number of weak periodic solutions of the following wave equation with boundary and periodic conditions:

$$
\begin{aligned}
& u_{t t}-u_{x x}=g(x, t, u), \\
& u(0, t)=u(\pi, t)=0, \\
& u(x, t+T)=u(x, t), \\
& u_{t}(x, 0)=u_{t}(x, T) \quad \forall x \in[0, \pi],
\end{aligned}
$$

where $T$ is a rational multiple of $\pi$. We assume that $g$ satisfies the following conditions:

(g1) $g \in C^{1}([0, \pi] \times R \times R, R)$ is $T$-periodic in $t$,

(g2) $g(x, t, 0)=0, g(x, t, \xi)=o(|\xi|)$ uniformly with respect to $x \in[0, \pi]$ and $t \in R$,

(g3) there exists $C>0$ such that $|g(x, t, \xi)|<C \forall x \in[0, \pi], t \in R, \xi \in R$.

Nonlinear problem of this type has been considered by many authors ( $c f .[1-5])$.

The purpose of this paper is to show the existence of $T$-periodic weak solutions of problem (1.1).

Our main result is as follows.

Theorem 1.1 Assume that g satisfies (g1)-(g3). Then problem (1.1) has at least one bounded solution.

For the proof of our main result, we approach the variational method and apply the critical point theory induced from the invariant subspaces and the invariant functional.

\section{Springer}

๑2013 Jung and Choi; licensee Springer. This is an Open Access article distributed under the terms of the Creative Commons Attribution License (http://creativecommons.org/licenses/by/2.0), which permits unrestricted use, distribution, and reproduction in any medium, provided the original work is properly cited. 
The outline of the proof of Theorem 1.1 is as follows. In Section 2, we introduce two Banach spaces $H$ and $E$ of functions satisfying some symmetry properties, stable by $A$ ( $A u=u_{t t}-$ $\left.u_{x x}\right), g$ such that the intersection of $H$ with the kernel of $A$ is reduced to 0 . The search of a solution of problem (1.1) in the space $H$ reduces the problem to a situation where $A^{-1}$ is a compact operator. In Section 3, we introduce a functional $I$ defined on $E$ whose critical points and weak solutions of (1.1) possess one-to-one correspondence. We prove that $I \in C^{1}(E, R)$ and satisfies the Palais-Smale condition. By a critical point theorem for indefinite functionals ( $c f$. [6]), we prove that there exists at least one solution of (1.1) which is bounded, and we prove Theorem 1.1.

\section{Invariant Banach space}

Let $\Omega=(0, \pi) \times(0, T)$; $T$ is a rational multiple of $\pi$

$$
T=\frac{2 \pi b}{a}
$$

where $a$ and $b$ are coprime integers. Let $\mathcal{A}$ be the operator defined by

$$
\begin{aligned}
& D(\mathcal{A})=\left\{u \in C^{2}(\bar{\Omega}) \mid u(0, t)=u(\pi, t)=0 \quad \forall t \in[0, T],\right. \\
& u(x, 0)=u(x, T) \quad \forall x \in[0, \pi], \\
& \left.u_{t}(x, 0)=u_{t}(x, T) \quad \forall x \in[0, \pi]\right\}, \\
& \mathcal{A} u=u_{t t}-u_{x x} .
\end{aligned}
$$

Let $A$ be the adjoint of $\mathcal{A}$ in $L^{2}(\Omega)$. We investigate the weak solutions of

$$
A u=g(x, t, u) .
$$

We note that the eigenvalues of $A$ are $\lambda_{j k}=j^{2}-\left(\frac{2 \pi k}{T}\right)^{2}, j=1,2, \ldots$ and $k=0,1,2, \ldots$, and the corresponding eigenfunctions are

$$
\sin j x \sin \frac{2 \pi k t}{T} \text { and } \sin j x \cos \frac{2 \pi k t}{T} .
$$

We also note that the set of functions $\sin j x \sin \frac{2 \pi k t}{T}, \sin j x \cos \frac{2 \pi k t}{T}$ is an orthogonal base for $L^{2}(\Omega)$. Let $u$ be a function of $L^{2}(\Omega)$. Then there exists one and only one function of $L^{2}([0, \pi] \times R)$ which is $T$-periodic in $t$ and equals $u$ on $\Omega$. We shall denote this function by $u$. Let us denote an element $u$, in $L^{2}(\Omega)$, by

$$
u=\sum_{j>0, k} u_{j k} \sin j x \exp i k \frac{a}{b} t
$$

with

$$
u_{j, k}=\bar{u}_{j,-k} .
$$

We assume that $b$ is even and $a$ is odd. Let $H$ be the closed subspace of $L^{2}(\Omega)$ defined by

$$
H=\left\{u \in L^{2}(\Omega) \mid u(x, t)=-u\left(x, t+\frac{T}{2}\right) \text { a.e. } x \in(0, \pi), t \in R\right\} .
$$


Then $H$ is invariant under shift: Let $h \in H$ and $\tau$ be a real number. If $v(x, t)=u(x, t+\tau)$, then $v \in H$. $H$ is invariant under $g$ : Let $u \in H$ such that $g(u) \in L^{2}(\Omega)$. Then $g(u) \in H$. Let $\tilde{u}(x, t)=u\left(x, t+\frac{T}{2}\right)$. Then

$$
u=\sum_{j>0, k} u_{j k}(-1)^{k} \sin j x \exp i k \frac{a}{b} t
$$

Therefore

$$
u \in H \quad \Longleftrightarrow \quad u_{j, k}=0 \quad \text { for any even } k
$$

Let $A_{1}$ be a linear operator of $H$ defined by

$$
\begin{aligned}
& D\left(A_{1}\right)=D(A) \cap H, \\
& A_{1} u=A u .
\end{aligned}
$$

Then $A_{1}$ is self-adjoint in $H$ and $H \cap N(A)=\{0\}$, where $N(A)$ is the kernel of $A$. In fact, let $u \in H \cap N(A)$. Then

$$
\begin{aligned}
& u=\sum u_{j, k} \sin j x \exp i k \frac{a}{b} t, \\
& j^{2}-\frac{k^{2} a^{2}}{b^{2}} \neq 0 \quad \Longrightarrow \quad u_{j, k}=0 .
\end{aligned}
$$

Let $j$ and $k$ be such that

$$
j^{2}-\frac{k^{2} a^{2}}{b^{2}}=0
$$

Since $b$ is even and $a$ is odd, $k$ is even. Using (2.1), we have $u_{j, k}=0$, and therefore $H \cap N(A)=$ $\{0\}$. The eigenvalues of $A_{1}$ are $j^{2}-\left(\frac{2 \pi k}{T}\right)^{2}$, where $j$ is odd and $k$ is even and $H \cap N(A)=\{0\}$. Given $u \in H$, we write

$$
u=\sum_{j>0} u_{j, k} \sin j x \exp i \frac{2 \pi k t}{T}, \quad j \text { is odd } k \text { is even }
$$

with

$$
u_{j, k}=\bar{u}_{j,-k} .
$$

Let

$$
\begin{aligned}
& E=\left\{u \in H\left|\sum_{j, k}\right| j^{2}-\left.\frac{a^{2} k^{2}}{b^{2}}|\cdot| u_{j, k}\right|^{2}<+\infty\right\}, \\
& (u, v)=\sum_{j, k}\left|j^{2}-\frac{a^{2} k^{2}}{b^{2}}\right| u_{j, k} \cdot \bar{v}_{j, k} \quad \text { for } u, v \in E,
\end{aligned}
$$


where $($,$) is a scalar product on E$. With this scalar product, $E$ is a Hilbert space with a norm

$$
\|u\|=(u, u)^{\frac{1}{2}}, \quad u \in E
$$

Let

$$
\|u\|_{r}=\left(\int_{\Omega}|u|^{r}\right)^{\frac{1}{r}}, \quad r \geq 1 .
$$

By the classical theorem of Riesz (cf. [7, p.525]), we have

$$
\|u\|_{r} \leq\left(\begin{array}{c}
\pi T \\
2
\end{array}\right)^{\frac{1}{r}}\left(\sum_{j, k}\left|u_{j, k}\right|^{r^{\prime}}\right)^{\frac{1}{r^{\prime}}}, \quad r \geq 2, \frac{1}{r}+\frac{1}{r^{\prime}}=1 .
$$

Since for every $\epsilon>o$

$$
\sum_{j \text { odd }, k \text { even }} \frac{1}{\left|j^{2}-\frac{a^{2} k^{2}}{b^{2}}\right|^{1+\epsilon}}<\infty
$$

it follows that for every $r \in[2,+\infty)$, there is $c_{r} \in R$ such that

$$
\|u\|_{r} \leq c_{r}\|u\| .
$$

Let

$$
\begin{aligned}
& E_{+}=\left\{u \mid u \in E, u_{j, k}=0 \text { if } j^{2}-\frac{a^{2} k^{2}}{b^{2}}<0\right\}, \\
& E_{-}=\left\{u \mid u \in E, u_{j, k}=0 \text { if } j^{2}-\frac{a^{2} k^{2}}{b^{2}}>0\right\} .
\end{aligned}
$$

Then $E=E_{+} \oplus E_{-}$. Let $P_{+}$be the orthogonal projection from $E$ onto $E_{+}$and $P_{-}$be the orthogonal projection from $E$ onto $E_{-}$. We can write $u=P_{+} u+P_{-} u$ for $u \in E$.

\section{Critical point theorem and the proof of Theorem 1.1}

Now, we are going to seek a function $u$ in $E$ such that

$$
A_{1} u=g(x, t, u) \text {. }
$$

We consider the associated functional of (3.1),

$$
\begin{aligned}
I(u) & =\frac{1}{2} \int_{\Omega}\left[-\left|u_{t}\right|^{2}+\left|u_{x}\right|^{2}\right] d x d t-\int_{\Omega} G(x, t, u) d x d t \\
& =\frac{1}{2}\left(\left\|P_{+} u\right\|^{2}-\left\|P_{-} u\right\|^{2}\right)-\int_{\Omega} G(x, t, u) d x d t,
\end{aligned}
$$

where

$$
G(x, t, u)=\int_{0}^{u} g(x, t, s) d s .
$$

By (g1), $I$ is well defined. 
We recall the critical point theorem for the indefinite functional (cf. [6]).

Let

$$
B_{r}=\{u \in H \mid\|u\| \leq r\}, \quad \partial B_{r}=\{u \in H \mid\|u\|=r\} .
$$

Theorem 3.1 (Critical point theorem for the indefinite functional) Let $E$ be a real Hilbert space with $E=E_{1} \oplus E_{2}$ and $E_{2}=E_{1}^{\perp}$. Suppose that $I \in C^{1}(E, R)$ satisfies (PS), and that

(I1) $I(u)=\frac{1}{2}(L u, u)+b u$, where $L u=L_{1} P_{1} u+L_{2} P_{2} u$ and $L_{i}: E_{i} \rightarrow E_{i}$ is bounded and self-adjoint, $i=1,2$,

(I2) $b^{\prime}$ is compact, and

(I3) there exist a subspace $\tilde{E} \subset E$ and sets $S \subset E, Q \subset \tilde{E}$ and constants $\alpha>\omega$ such that

(i) $S \subset E_{1}$ and $\left.I\right|_{S} \geq \alpha$,

(ii) $Q$ is bounded and $\left.I\right|_{\partial Q} \leq \omega$,

(iii) $S$ and $\partial Q$ link.

Then I possesses a critical value $c \geq \alpha$.

The eigenvalues of $A_{1}$ are $\lambda_{j k}=j^{2}-\left(\frac{2 \pi k}{T}\right)^{2}$, where $j$ is odd and $k$ is even, $j=1,2, \ldots$ and $k=0,1,2, \ldots$. Thus $A_{1}^{-1}$ is a compact operator.

It is convenient for the following to rearrange the eigenvalues $\lambda_{j k}$, where $j$ is odd and $k$ is even, by increasing magnitude: from now on we denote by $\left(\eta_{i}^{-}\right)_{i \geq 1}=\lambda_{j k}<0$ the sequence of negative eigenvalues, by $\left(\eta_{i}^{+}\right)_{i \geq 1}=\lambda_{j k}>0$ the sequence of positive ones, so that

$$
\cdots \leq \eta_{i}^{-} \leq \cdots \leq \eta_{2}^{-} \leq \eta_{1}^{-}<\eta_{1}^{+} \leq \eta_{2}^{+} \leq \cdots \leq \eta_{i}^{+} \leq \cdots .
$$

We note that each eigenvalue has a finite multiplicity and that $\eta_{i}^{-} \rightarrow-\infty, \eta_{i}^{+} \rightarrow+\infty$ as $i \rightarrow \infty$.

We shall show that the functional $I$ satisfies the geometrical assumptions of the critical point theorem for the indefinite functional.

By the following lemma, $I(u) \in C^{1}(E, R)$ and the solutions of (3.1) coincide with the nonzero critical points of $I(u)$.

Lemma 3.1 Assume that $g$ satisfies (g1)-(g3). Then I(u) is continuous and Fréchet differentiable in $E$ with the Fréchet derivative

$$
\begin{aligned}
I^{\prime}(u) h & =\int_{\Omega}\left[-u_{t} \cdot h_{t}+u_{x} \cdot h_{x}-g(x, t, u) h\right] d x d t \\
& =\left(P_{+} u, P_{+} h\right)-\left(P_{-} u, P_{-} h\right)-\int_{\Omega} g(x, t, u) h d x d t
\end{aligned}
$$

for all $h \in E$. Moreover, if we set

$$
F(u)=\int_{\Omega} G(x, t, u) d x d t,
$$

then $F^{\prime}(u)$ is continuous with respect to weak convergence, $F^{\prime}(u)$ is compact, and

$$
F^{\prime}(u) h=\int_{\Omega} g(x, t, u) h d x d t \quad \text { for all } h \in E .
$$

This implies that $I \in C^{1}(E, R)$ and $F(u)$ is weakly continuous. 
For the proof of Lemma 3.1, refer to [6].

Lemma 3.2 Assume that $g$ satisfies (g1)-(g3). The problem

$$
\begin{aligned}
& u_{t t}-u_{x x} \quad \text { in } E, \\
& u(0, t)=u(\pi, t)=0, \\
& u(x, t+T)=u(x, t), \\
& u_{t}(x, 0)=u_{t}(x, T) \quad \forall x \in[0, \pi]
\end{aligned}
$$

has only a trivial solution.

Proof Let $A u=u_{t t}-u_{x x}$. Since $H \cap N(A)=\{0\}, E \cap N(A)=\{0\}$. Thus (3.4) has only a trivial solution.

Lemma 3.3 Assume that $g$ satisfies (g1)-(g3). Then I(u) satisfies the Palais-Smale condition: If for a sequence $\left(u_{m}\right), I\left(u_{m}\right)$ is bounded from above and $I^{\prime}\left(u_{m}\right) \rightarrow 0$ as $m \rightarrow \infty$, then $\left(u_{m}\right)$ has a convergent subsequence.

Proof Let $\left(u_{m}\right)$ be a sequence with $I\left(u_{m}\right) \leq M$ and $I^{\prime}\left(u_{m}\right) \rightarrow 0$ as $m \rightarrow \infty$. It suffices to show that $\left(u_{m}\right)$ is bounded. By contradiction, we suppose that $\left\|u_{m}\right\| \rightarrow \infty$ as $m \rightarrow \infty$. Let $w_{m}=\frac{u_{m}}{\left\|u_{m}\right\|}$. Then $\left\|w_{m}=1\right\|$ and $w_{m}$ converges weakly to an element, say $w$. Then we have

$$
\frac{M}{\left\|u_{m}\right\|^{2}} \leq \frac{I\left(u_{m}\right)}{\left\|u_{m}\right\|^{2}}=\frac{1}{2} \int_{\Omega}\left[-\left|\left(w_{m}\right)_{t}\right|^{2}+\left|\left(w_{m}\right)_{x x}\right|^{2}\right]-\int_{\Omega} \frac{G\left(x, t, u_{m}\right)}{\left\|u_{m}\right\|^{2}}
$$

Since $G(x, t, u)$ is bounded, letting $m \infty$ in (3.5), we have

$$
0 \leq \lim P_{+} w_{m}-\lim P_{-} w_{m}=\int_{\Omega}\left[-\left|w_{t}\right|^{2}+\left|w_{x x}\right|^{2}\right] .
$$

On the other hand, we have

$$
0 \longleftarrow \frac{I^{\prime}\left(u_{m}\right)}{\left\|u_{m}\right\|^{2}}=\int_{\Omega}\left[-\left|\left(w_{m}\right)_{t}\right|^{2}+\left|\left(w_{m}\right)_{x x}\right|^{2}\right]-\int_{\Omega} \frac{g\left(x, t, u_{m}\right)}{\left\|u_{m}\right\|^{2}} .
$$

Letting $m \rightarrow \infty$ in (3.7), we have

$$
0=\lim _{m \rightarrow \infty}\left\|P_{+} w_{m}\right\|^{2}-\lim _{m \rightarrow \infty}\left\|P_{-} w_{m}\right\|^{2}=\int_{\Omega}\left[-\left|w_{t}\right|^{2}+\left|w_{x x}\right|^{2}\right]=\left\|P_{+} w\right\|^{2}-\left\|P_{-} w\right\|^{2} .
$$

By (3.6) and (3.8), $\lim _{m \rightarrow \infty}\left\|P_{+} w_{m}\right\|^{2}=\left\|P_{+} w\right\|^{2}$ and $\lim _{m \rightarrow \infty}\left\|P_{-} w_{m}\right\|^{2}=\left\|P_{-} w\right\|^{2}$. Thus $\lim _{m \rightarrow \infty}\left\|w_{m}\right\|=\|w\|$, so $w_{m}$ converges strongly to $w$ and by (3.8), $w$ is a weak solution of the problem

$$
w_{t t}-w_{x x}=0 \quad \text { in } E .
$$

By Lemma 3.2, $w=0$, which is absurd for the fact that $\|w\|=1$. Thus $\left(u_{m}\right)$ is bounded. 
Let

$$
Q=\left(\bar{B}_{R} \cap E_{-}\right) \oplus\left\{r e \mid e \in \partial B_{1} \cap E_{+} 0<r<R\right\} .
$$

Lemma 3.4 Assume that $g$ satisfies the conditions (g1)-(g3). There exist a subspace $\tilde{E} \subset E$ and sets $\partial B_{\rho} \subset E, Q \subset \tilde{E}$ and constants $\rho>0$ and $\alpha>0$ such that

(i) $\partial B_{\rho} \subset E_{+}$and $\left.I\right|_{\partial B_{\rho}} \geq \alpha$,

(ii) there are $e \in \partial B_{1} \cap E_{+}$and $R>\rho$ such that if $Q=\left(\bar{B}_{R} \cap E_{-}\right) \oplus\{r e \mid 0<r<R\}$, then $\left.I\right|_{\partial Q} \leq 0$,

(iii) there exists $u_{0} \in E \backslash Q$ such that $\left\|u_{0}\right\|>R$ and $I\left(u_{0}\right) \leq 0$,

(iv) $\partial B_{\rho}$ and $\partial Q$ link.

Proof (i) Let $u \in E_{+}$. Since $G(x, t, u)$ is bounded, there exists a constant $C>0$ such that

$$
\begin{aligned}
I(u) & =\frac{1}{2}\left\|P_{+} u\right\|^{2}-\frac{1}{2}\left\|P_{-} u\right\|^{2}-\int_{\Omega} G(x, t, u) \\
& \geq \frac{1}{2}\left\|P_{+} u\right\|^{2}-C
\end{aligned}
$$

for $C>0$. Then there exist a constant $\rho>0$ and a constant $\alpha>0$ such that if $u \in \partial B_{\rho} \cap E_{+}$, then $I(u) \geq \alpha$.

(ii) Let us choose an element $e \in B_{1} \cap E_{+}$. Let $u \in\left(\bar{B}_{r} \cap E_{-}\right) \oplus\{r e \mid 0<r\}$. Then $u=v+w$, $v \in B_{r} \cap E_{-}, w=r e$. We note that

$$
\text { if } v \in B_{r} \cap E_{-} \text {, then } \int_{\Omega}\left[-\left|v_{t}\right|^{2}+\left|v_{x}\right|^{2}\right] d x d t \leq \eta_{i}^{-}\|v\|_{L^{2}(\Omega)}^{2}<0 .
$$

Thus we have

$$
\begin{aligned}
I(u) & =\frac{1}{2} r^{2}-\frac{1}{2}\|v\|^{2}-\int_{\Omega} G(x, t, v+r e) \\
& \leq \frac{1}{2} r^{2}+\frac{1}{2} \eta_{1}^{-}\|v\|_{L^{2}(\Omega)}^{2}+C_{1}
\end{aligned}
$$

for $C_{1}>0$. Then there exists $R>\rho>0$ such that if $u \in Q=\left(\bar{B}_{R} \cap E_{-}\right) \oplus\{r e \mid 0<r<R\}$, then $\left.I(u)\right|_{\partial Q} \leq 0$.

(iii) Let us choose $u_{0} \in E \backslash Q$. By (ii), $I\left(u_{0}\right) \leq 0$.

(iv) $S$ and $Q$ link at the set $\{e\}$.

Proof of Theorem 1.1 By Lemma 3.1, $I(u)$ is continuous and Fréchet differentiable in $E$. By Lemma 3.3, the functional $I$ satisfies the (PS) condition. We note that $I(0)=0$. By Lemma 3.4, there are constants $\rho>0, \alpha>0$ and a bounded neighborhood $B_{\rho}$ of 0 such that $\left.I\right|_{\partial B_{\rho} \cap E_{+}} \geq \alpha$, and there are $e \in \partial B_{1} \cap E_{+}$and $R>\rho$ such that if $Q=\left(\bar{B}_{R} \cap E_{-}\right) \oplus\{r e \mid 0<r<R\}$. Let us set

$$
\Gamma=\{\gamma \in C(\bar{Q}, H) \mid \gamma=\text { id on } \partial Q\} .
$$

By Theorem 3.1, $I$ possesses a critical value $c \geq \alpha$. Moreover, $c$ can be characterized as

$$
c=\inf _{\gamma \in \Gamma} \max _{u \in Q} I(\gamma(u))
$$


Thus we prove that $I$ has at least one nontrivial critical point, we denote by $\tilde{u}$ a critical point of $I$ such that $I(\tilde{u})=c$. We claim that $c$ is bounded. In fact, we have

$$
c \leq \max _{0 \leq \tau \leq 1} I\left(\tau u_{0}\right)
$$

and by (g3),

$$
\begin{aligned}
I\left(\tau u_{0}\right) & =\tau^{2}\left(\frac{1}{2}\left\|P_{+} u_{0}\right\|^{2}-\frac{1}{2}\left\|P_{-} u_{0}\right\|^{2}\right)-\int_{\Omega} G\left(x, t, \tau u_{0}\right) d x d t \\
& \leq \tau^{2}\left\|u_{0}\right\|^{2}-\int_{\Omega} G\left(x, t, \tau u_{0}\right) d x d t \\
& \leq C_{1} \tau^{2}\left\|u_{0}\right\|^{2}+C_{1}
\end{aligned}
$$

for some constant $C_{1}>0$. Since $0 \leq \tau \leq 1, c$ is bounded:

$$
c<\tilde{C} .
$$

We claim that $\tilde{u}$ is bounded. In fact, by contradiction, $\tilde{u}_{t t}+\tilde{u}_{x x}=g(x, t, \tilde{u})$ and, for any $K>0$, $\max _{\Omega}|\tilde{u}(x, t)|>K$ imply that

$$
c=I(\tilde{u})=\frac{1}{2}\left(\left\|P_{+} \tilde{u}\right\|^{2}-\left\|P_{-} \tilde{u}\right\|^{2}\right)-\int_{\Omega} G(x, t, \tilde{u}) d x d t
$$

is not bounded, which is absurd to the fact that $c=I(\tilde{u})$ is bounded. Thus $\tilde{u}$ is bounded, so (1.1) has at least one bounded weak solution, and hence we prove Theorem 1.1.

\section{Competing interests}

The authors did not provide this information.

\section{Authors' contributions}

The authors did not provide this information.

\section{Author details}

${ }^{1}$ Department of Mathematics, Kunsan National University, Kunsan, 573-701, Korea. ${ }^{2}$ Department of Mathematics Education, Inha University, Incheon, 402-751, Korea.

\section{Acknowledgements}

This work (Choi) was supported by Basic Science Research Program through the National Research Foundation of Korea (NRF) funded by the Ministry of Education, Science and Technology (KRF-2013010343).

Received: 27 June 2013 Accepted: 25 October 2013 Published: 25 Nov 2013

\section{References}

1. Amann, H, Zehnder, E: Multiple periodic solutions for a class of nonlinear-autonomous wave equations. Houst. J. Math. 7, 147-174 (1981)

2. Brezis, H, Coron, JM: Periodic solutions of nonlinear wave equations and Hamiltonian systems. Am. J. Math. 103 559-570 (1981)

3. Brezis, H, Nirenberg, L: Forced vibrations for a nonlinear wave equation. Commun. Pure Appl. Math. 31, 1-30 (1978)

4. Choi, QH, Jung, T: An application of a variational reduction method to a nonlinear wave equation. J. Differ. Equ. 7, 390-410 (1995)

5. Rabinowitz, PH: Free vibrations for a semilinear wave equation. Commun. Pure Appl. Math. 31, 31-68 (1968)

6. Benci, V, Rabinowitz, PH: Critical point theorems for indefinite functionals. Invent. Math. 52, 241-273 (1979)

7. Fadell, ER, Rabinowitz, PH: Generalized cohomological index theories for the group actions with an application to bifurcation questions for Hamiltonian systems. Invent. Math. 45, 139-174 (1978)

Cite this article as: Jung and Choi: Bounded solutions for the nonlinear wave equation. Boundary Value Problems 2013, 2013:257 